\title{
A Spectral Regularization Method for a Cauchy Problem of the Modified Helmholtz Equation
}

\author{
Ailin Qian, Jianfeng Mao, and Lianghua Liu \\ School of Mathematics and Statistics, Xianning College, Xianning, Hubei 437100, China \\ Correspondence should be addressed to Ailin Qian, junren751113@126.com
}

Received 15 December 2009; Accepted 9 May 2010

Academic Editor: Salim Messaoudi

Copyright (c) 2010 Ailin Qian et al. This is an open access article distributed under the Creative Commons Attribution License, which permits unrestricted use, distribution, and reproduction in any medium, provided the original work is properly cited.

We consider the Cauchy problem for a modified Helmholtz equation, where the Cauchy data is given at $x=1$ and the solution is sought in the interval $0<x<1$. A spectral method together with choice of regularization parameter is presented and error estimate is obtained. Combining the method of lines, we formulate regularized solutions which are stably convergent to the exact solutions.

\section{Introduction}

The Cauchy problem for Helmholtz equation arises from inverse scattering problems. Specific backgrounds can be seen in the existing literature; we can refer to [1-6] and so forth. A number of numerical methods for stabilizing this problem are developed. Several boundary element methods combined with iterative, conjugate gradient, Tikhonov regularization, and singular value decomposition methods are compared in [6]. Cauchy problem for elliptic equations is well known to be severely ill-posed [7]; that is, the solution does not depend continuously on the boundary data, and small errors in the boundary data can amplify the numerical solution infinitely; hence it is impossible to solve Cauchy problem of Helmholtz equation by using classical numerical methods and it requires special techniques, for example, regularization methods. Although theoretical concepts and computational implementation related to the Cauchy problem of Helmholtz equation have been discussed by many authors [8-11], there are many open problems deserved to be solved. For example, many authors have considered the following Cauchy problem of the Helmholtz equation [8-11]:

$$
\begin{gathered}
u_{x x}+u_{y y}-k^{2} u(x, y)=0, \quad 0<x<1,-\infty<y<+\infty, \\
u(1, y)=g(y), \quad-\infty<y<+\infty \\
u_{x}(1, y)=0, \quad-\infty<y<+\infty .
\end{gathered}
$$


However, the boundary condition $u_{x}(1, y)=0$ is very strict. If the boundary condition is replaced by $u_{x}(1, y)=h(y)$, where $h(y)$ is a function of $y$, then their methods cannot be applied easily. Therefore, in this paper, we consider the following Cauchy problem for the Helmholtz equation:

$$
\begin{gathered}
u_{x x}+u_{y y}-k^{2} u(x, y)=0, \quad 0<x<1,-\infty<y<+\infty, \\
u(1, y)=g(y), \quad-\infty<y<+\infty, \\
u_{x}(1, y)=h(y), \quad-\infty<y<+\infty .
\end{gathered}
$$

We want to seek the solution in the interval $(0,1)$ from the Cauchy data pairs $(g, h)$ located at $x=1$. Of course, since $g$ and $h$ are assumed to be measured, there must be measurement errors, and we would actually have noisy data function $g_{\delta}, h_{\delta} \in L^{2}(\mathbb{R})$, for which the measurement errors $\left\|h-h_{\mathcal{\delta}}\right\|$ and $\left\|g-g_{\delta}\right\|$ are small. Here and in the following sections, $\|\cdot\|$ denotes the $L^{2}(\mathbb{R})$ norm. Thus $(1.2)$ is a noncharacteristic Cauchy problem with appropriate Cauchy data $\left[u, u_{x}\right]$ given on the line $x=1$.

However, the ill-posedness is caused by high frequency. By introducing a "cutoff" frequency we can obtain a well-posed problem. This method has been used for solving inverse heat conduction problem [12], and sideways heat equation [13], sideways parabolic equation [14]. An error estimate for the proposed method can be found in Section 2. The implementation of the numerical method is explained.

\section{Regularization and Error Estimate}

First, let $\widehat{u}$ be the Fourier transform of $u$ :

$$
\widehat{u}(x, \xi)=\frac{1}{\sqrt{2 \pi}} \int_{-\infty}^{+\infty} u(x, y) e^{-i \xi y} d y, \quad \xi \in \mathbb{R} .
$$

Taking Fourier transformation for (1.2), we have a family of problems parameterized by $\xi$ :

$$
\begin{gathered}
\widehat{u}_{x x}(x, \xi)-\xi^{2} \widehat{u}(x, \xi)-k^{2} \widehat{u}(x, \xi)=0, \quad 0<x<1, \xi \in \mathbb{R}, \\
\widehat{u}(1, \xi)=\widehat{g}(\xi), \quad \xi \in \mathbb{R}, \\
\widehat{u}_{x}(1, \xi)=\widehat{h}(\xi), \quad \xi \in \mathbb{R} .
\end{gathered}
$$

The solution can easily be verified to be

$$
\widehat{u}(x, \xi)=-\frac{\widehat{h}(\xi)}{\sqrt{\xi^{2}+k^{2}}} \sinh \left((1-x) \sqrt{\xi^{2}+k^{2}}\right)+\widehat{g}(\xi) \cosh \left((1-x) \sqrt{\xi^{2}+k^{2}}\right) .
$$


Following the idea of Fourier method, we consider (2.2) only for $|\xi|<\xi_{c}$ by cutting off high frequency and define a regularized solution:

$$
\widehat{u}_{c}(x, \xi)=x_{c}(\xi) \widehat{u}(x, \xi)
$$

where $x_{c}$ is the characteristic function of the interval $\left[-\xi_{c}, \xi_{c}\right]$. The solution $u_{c}(x, y)$ can be found by using the inverse Fourier transform. Define the regularized solution with measured data $\left[g_{\delta}, h_{\delta}\right]$ by $u_{c}^{\delta}$. The difference between the exact solution $u(x, y)$ and the regularized solution $u_{c}^{\delta}(x, y)$ can be divided into two parts:

$$
\left\|u(x, \cdot)-u_{c}^{\delta}(x, \cdot)\right\| \leq\left\|u-u_{c}\right\|+\left\|u_{c}-u_{c}^{\delta}\right\|=R_{a}+R_{b},
$$

where

$$
R_{a}^{2}=\int_{|\xi| \xi \xi_{c}}|\widehat{u}(x, \xi)|^{2} d \xi, \quad R_{b}^{2}=\int_{-\xi_{c}}^{\xi_{c}}\left|\widehat{u}_{c}-\widehat{u}_{c}^{\delta}\right|^{2} d \xi .
$$

We rewrite (2.2) as a system of ordinary differential equations:

$$
\left(\begin{array}{c}
\sqrt{\xi^{2}+k^{2}} \widehat{u} \\
\widehat{u}_{x}
\end{array}\right)_{x}=\left(\begin{array}{cc}
0 & \sqrt{\xi^{2}+k^{2}} \\
\sqrt{\xi^{2}+k^{2}} & 0
\end{array}\right)\left(\begin{array}{c}
\sqrt{\xi^{2}+k^{2}} \widehat{u} \\
\widehat{u}_{x}
\end{array}\right), \quad 0 \leq x \leq 1 .
$$

Letting $U(x)=\left(\sqrt{\xi^{2}+k^{2}} \widehat{u} \widehat{u}_{x}\right)^{T}$, we can rewrite (2.7) as

$$
U_{x}(x)=A U(x), \quad 0 \leq x \leq 1, \quad U(1)=\left(\begin{array}{c}
\sqrt{\xi^{2}+k^{2}} \hat{g} \\
\hat{h}
\end{array}\right)
$$

where the matrix $A$ is the one in (2.7). The reason for using $\sqrt{\xi^{2}+k^{2}} \widehat{u}$ instead of $\widehat{u}$ in the definition of $U(x)$ is that with this choice, the matrix $A$ is normal and hence diagonalized by a unitary matrix. The eigenvalues of $A$ are $\lambda_{1,2}= \pm \sqrt{\xi^{2}+k^{2}}$. Thus we can factorize $A$ as

$$
A=X \Lambda X^{H}, \quad \Lambda=\left(\begin{array}{cc}
\sqrt{\xi^{2}+k^{2}} & 0 \\
0 & -\sqrt{\xi^{2}+k^{2}}
\end{array}\right),
$$

where $X$ is a unitary matrix, $X^{H} X=I$; it follows that the solution of the system (2.8) can be written as

$$
U(x)=X e^{\Lambda(x-1)} X^{H} U(1) .
$$

Since $X$ is unitary, $\|X\|=1$ and therefore

$$
\|U(x)\|_{2}^{2} \leq\left\|e^{\Lambda(x-1)}\right\|_{2}^{2} \cdot\|U(1)\|_{2}^{2}
$$


where $\|\cdot\|_{2}$ denotes both the Euclidean norm in the complex vector space $C^{2}$ and the subordinate matrix norm, remembering $U=\left(\sqrt{\xi^{2}+k^{2}} \widehat{u} \widehat{u}_{x}\right)^{\mathrm{T}}$ in $(2.11)$, we obtain

$$
\left|\sqrt{\xi^{2}+k^{2}} \widehat{u}\right|^{2}+\left|\widehat{u}_{x}\right|^{2} \leq e^{2 \sqrt{\xi^{2}+k^{2}}(1-x)}\left(\left|\sqrt{\xi^{2}+k^{2}} \widehat{u}(1, \xi)\right|^{2}+\left|\widehat{u}_{x}(1, \xi)\right|^{2}\right) .
$$

This inequality is valid for all $\xi \in R$ and we can integrate over $\xi$ and use the Parseval theorem to obtain estimates for $u$ and $u_{x}$ in the $L^{2}(\mathbb{R})$-norm. First we will prove a bound on the difference between any two regularized solutions (2.4). We have errors in the measured $g(y)$ and $h(y)$. These two cases are treated separately.

Lemma 2.1. Assume that one has two regularized solutions $u_{1}$ and $u_{2}$ defined by (2.4), with the Cauchy data $\left[g, h_{1}\right]$ and $\left[g, h_{2}\right]$. Then

$$
\left\|u_{1}(x, \cdot)-u_{2}(x, \cdot)\right\| \leq \frac{e^{\sqrt{\xi_{c}^{2}+k^{2}}(1-x)}-1}{\sqrt{\xi_{c}^{2}+k^{2}}}\left\|h_{1}-h_{2}\right\| .
$$

Proof. The function $w=\widehat{u}_{1}-\widehat{u}_{2}$ satisfies the differential equation; thus $w$ solves (2.2), with initial data given by

$$
w(1, \cdot)=0, \quad w_{x}(1, \cdot)=\left(\widehat{h}_{1}-\widehat{h}_{2}\right) X_{c}
$$

and thus $w$ satisfies inequality(2.12). We have

$$
\left\|w_{x}(x, \xi)\right\|^{2} \leq e^{2 \sqrt{\xi^{2}+k^{2}}(1-x)}\left|w_{x}(1, \xi)\right|^{2} \leq e^{2 \sqrt{\xi_{c}^{2}+k^{2}}(1-x)}\left|\widehat{h}_{1}-\widehat{h}_{2}\right|^{2} .
$$

If $0 \leq z \leq 1$, then

$$
w(z, \xi)=-\int_{z}^{1} w_{x}(x, \xi) d x+w(1, \xi)=-\int_{z}^{1} w_{x}(x, \xi) d x
$$

holds. By inserting (2.15) into (2.16) we get

$$
\begin{aligned}
|w(z, \xi)| & =\left|\int_{z}^{1} w_{x}(x, \xi) d x\right| \leq \int_{z}^{1}\left|w_{x}\right| d x \leq \int_{z}^{1} e^{\sqrt{\xi_{c}^{2}+k^{2}}(1-x)}\left|w_{x}(1, \xi)\right| d x \\
& =\frac{e^{\sqrt{\xi_{c}^{2}+k^{2}}(1-z)}-1}{\sqrt{\xi_{c}^{2}+k^{2}}} \cdot\left|w_{x}(1, \xi)\right| .
\end{aligned}
$$


If we insert $w=\widehat{u}_{1}-\widehat{u}_{2}$ and integrate over $\xi$, then we obtain

$$
\int_{-\infty}^{+\infty}\left(\widehat{u}_{1}-\widehat{u}_{2}\right)^{2} d \xi \leq\left(\frac{e^{\sqrt{\xi_{c}^{2}+k^{2}}(1-x)}-1}{\sqrt{\xi_{c}^{2}+k^{2}}}\right)^{2} \cdot \int_{-\infty}^{+\infty}\left|\widehat{h}_{1}(\xi)-\widehat{h}_{2}(\xi)\right|^{2} d \xi
$$

Thus (2.13) holds.

Lemma 2.2. Assume that one has two regularized solutions $u_{1}$ and $u_{2}$ defined by (2.4), with the Cauchy data $\left[g_{1}, h\right]$ and $\left[g_{2}, h\right]$. Then

$$
\left\|u_{1}(x, \cdot)-u_{2}(x, \cdot)\right\| \leq e^{\sqrt{\xi_{c}^{2}+k^{2}}(1-x)}\left\|g_{1}-g_{2}\right\| .
$$

Proof. The function $w=\widehat{u}_{1}-\widehat{u}_{2}$ satisfies the differential equation, with initial data given by

$$
w(1, \xi)=\left(\widehat{g}_{1}-\widehat{g}_{2}\right) X_{c}, \quad w_{x}(1, \xi)=0
$$

and thus inequality (2.12) holds. It follows that

$$
\left\|\sqrt{\xi^{2}+k^{2}} w(x, \xi)\right\|^{2} \leq e^{2 \sqrt{\xi^{2}+k^{2}}(1-x)}|\xi w(1, \xi)|^{2}
$$

and thus, for $\xi^{2}+k^{2} \neq 0$,

$$
\|w(x, \xi)\|^{2} \leq e^{2 \sqrt{\xi^{2}+k^{2}}(1-x)}|w(1, \xi)|^{2}
$$

By integrating over the interval $\left[-\xi_{c}, \xi_{c}\right]$, we get

$$
\int_{-\xi_{c}}^{\xi_{c}}|w(x, \xi)|^{2} d \xi \leq \int_{-\xi_{c}}^{\xi_{c}} e^{2 \sqrt{\xi^{2}+k^{2}}(1-x)}|w(1, \xi)|^{2} d \xi \leq e^{2 \sqrt{\xi_{c}^{2}+k^{2}}(1-x)} \int_{-\xi_{c}}^{\xi_{c}}|w(1, \xi)|^{2} d \xi .
$$

Since $w=\widehat{u}_{1}-\widehat{u}_{2}$ is equal to zero outside the interval $\left[-\xi_{c}, \xi_{c}\right]$, we can extend the integrals. Inserting $w=\widehat{u}_{1}-\widehat{u}_{2}$, we get

$$
\int_{-\infty}^{+\infty}\left(\widehat{u}_{1}-\widehat{u}_{2}\right)^{2} d \xi \leq e^{2 \sqrt{\xi_{c}^{2}+k^{2}}(1-x)} \cdot \int_{-\infty}^{+\infty}\left|\widehat{g}_{1}-\widehat{g}_{2}\right|^{2} d \xi
$$

This is precisely (2.19).

Next we prove that, for the regularized problem, we have stable dependence of the data. By using the two previous lemmas, we get the following. 
Lemma 2.3 (stability). Assume that $u_{c}$ is the regularized solution (2.4), with exact data $(g, h)$, and $u_{c}^{\delta}$ is the regularized solution with noisy data $\left[g_{\delta}, h_{\delta}\right]$; then

$$
\left\|u_{c}-u_{c}^{\delta}\right\| \leq e^{\sqrt{\xi_{c}^{2}+k^{2}}(1-x)}\left(\left\|g-g_{\delta}\right\|+\frac{\left\|h-h_{\delta}\right\|}{\sqrt{\xi_{c}^{2}+k^{2}}}\right)
$$

Proof. Let $u_{1}$ be a regularized solution defined by (2.4), with data $\left[g, h_{\delta}\right]$. Then by Lemma 2.1,

$$
\left\|u_{c}-u_{1}\right\| \leq \frac{e^{\sqrt{\xi_{c}^{2}+k^{2}}(1-x)}-1}{\sqrt{\xi_{c}^{2}+k^{2}}}\left\|h-h_{\delta}\right\| \leq \frac{e^{\sqrt{\xi_{c}^{2}+k^{2}}(1-x)}}{\sqrt{\xi_{c}^{2}+k^{2}}}\left\|h-h_{\delta}\right\| .
$$

Using Lemma 2.2, we have

$$
\left\|u_{1}-u_{c}^{\delta}\right\| \leq e^{\sqrt{\xi_{c}^{2}+k^{2}}(1-x)}\left\|g-g_{\delta}\right\|
$$

By the triangle inequality,

$$
\left\|u_{c}-u_{c}^{\delta}\right\| \leq\left\|u_{c}-u_{1}\right\|+\left\|u_{1}-u_{c}^{\delta}\right\| \leq e^{\sqrt{\xi_{c}^{2}+k^{2}}(1-x)}\left(\left\|g-g_{\delta}\right\|+\frac{\left\|h-h_{\delta}\right\|}{\sqrt{\xi_{c}^{2}+k^{2}}}\right) .
$$

This completes the proof.

By Lemma 2.3 the regularized solution depends continuously on the data. Next we derive a bound on the truncation error when we neglect frequencies $|\xi| \geq \xi_{c}$ in (2.3). So far we have not used any information about $\widehat{u}$ for $x>1$; we, assume that the Helmholtz equation $u_{x x}+u_{y y}-k^{2} u=0$ is valid in a large interval $0<x<L, L>1$. By imposing a priori bounds on the solution at $x=0$ and at $x>1$, we obtain an estimate of the difference between the exact solution $u$ and a regularized solution $u_{c}$ with "cutoff" level $\xi_{c}$. This is a convergence result in the sense that $u_{c} \rightarrow u$ as $\xi_{c} \rightarrow \infty$ for the case of exact data $[g, h]$. The following estimate holds.

Lemma 2.4 (convergence). Suppose that $\widehat{u}$ is the solution of the problem (2.2), and that the Helmholtz equation is valid for $0<x<L$. Then the difference between $\widehat{u}$ and a regularized solution $\widehat{u}_{c}$ can be estimated:

$$
\left\|\widehat{u}(x, \cdot)-\widehat{u}_{c}(x, \cdot)\right\| \leq C\left(e^{-x \sqrt{\xi_{c}^{2}+k^{2}}}\|u(0, \cdot)\|+e^{(x-L)} \sqrt{\xi_{c}^{2}+k^{2}}\|u(L, \cdot)\|\right)
$$

where the constant $C$ is defined by

$$
C=\sup _{\substack{0 \leq x \leq L, \xi \neq 0}} \frac{1-e^{-2 x \sqrt{\xi^{2}+k^{2}}}}{1-e^{-2 L \sqrt{\xi^{2}+k^{2}}}}<2
$$


Proof. The solution of (2.2) can be written in the floowing form:

$$
\widehat{u}=A e^{x \sqrt{\xi^{2}+k^{2}}}+B e^{-x \sqrt{\xi^{2}+k^{2}}}
$$

where $A$ and $B$ can be determined from the boundary conditions:

$$
\left(\begin{array}{cc}
1 & 1 \\
e^{L \sqrt{\xi^{2}+k^{2}}} & e^{-L \sqrt{\xi^{2}+k^{2}}}
\end{array}\right)\left(\begin{array}{l}
A \\
B
\end{array}\right)=\left(\begin{array}{c}
\widehat{u}(0, \xi) \\
\widehat{u}(L, \xi)
\end{array}\right)
$$

Solving for $A$ and $B$ we find that the solution can be written:

$$
\widehat{u}(x, \xi)=\varphi(L-x, \xi) \widehat{u}(0, \xi)+\varphi(x, \xi) \widehat{u}(L, \xi),
$$

where

$$
\varphi(x, \xi)=\frac{e^{x \sqrt{\xi^{2}+k^{2}}}-e^{-x \sqrt{\xi^{2}+k^{2}}}}{e^{L \sqrt{\xi^{2}+k^{2}}}-e^{-L \sqrt{\xi^{2}+k^{2}}}}=e^{(x-L) \sqrt{\xi^{2}+k^{2}}} \frac{1-e^{-2 x \sqrt{\xi^{2}+k^{2}}}}{1-e^{-2 L \sqrt{\xi^{2}+k^{2}}}} .
$$

We make the observation that

$$
|\varphi(x, \xi)| \leq C e^{(x-L) \sqrt{\xi^{2}+k^{2}}}
$$

and that

$$
|\varphi(L-x, \xi)| \leq C e^{-x \sqrt{\xi^{2}+k^{2}}}
$$

Using the expression (2.33) and the triangle inequality, we get

$$
\left\|\widehat{u}-\widehat{u}_{c}\right\| \leq\left\|\varphi(L-x, \cdot)\left[\widehat{u}(0, \cdot)-\widehat{u}_{c}(0, \cdot)\right]\right\|+\left\|\varphi(x, \cdot)\left[\widehat{u}(L, \cdot)-\widehat{u}_{c}(L, \cdot)\right]\right\| .
$$

The first term on the right-hand side satisfies

$$
\begin{aligned}
\left\|\varphi(L-x, \cdot)\left[\widehat{u}(0, \cdot)-\widehat{u}_{c}(0, \cdot)\right]\right\|^{2} & \leq \int_{|\xi|>\xi_{c}} C^{2} e^{-2 x \sqrt{\xi^{2}+k^{2}}}|\widehat{u}(0, \xi)|^{2} d \xi \\
& \leq C^{2} e^{-2 x \sqrt{\xi_{c}^{2}+k^{2}}} \int_{|\xi| \xi \xi_{c}}|\widehat{u}(0, \xi)|^{2} d \xi \\
& \leq C^{2} e^{-2 x \sqrt{\xi_{c}^{2}+k^{2}}}\|\widehat{u}(0, \xi)\|^{2}
\end{aligned}
$$


Similarly, the second term can be estimated:

$$
\left\|\varphi(x, \cdot)\left[\widehat{u}(L, \cdot)-\widehat{u}_{c}(L, \cdot)\right]\right\|^{2} \leq C^{2} e^{-2(L-x) \sqrt{\xi_{c}^{2}+k^{2}}} \cdot\|\widehat{u}(L, \xi)\|^{2}
$$

By combining these two expressions, we have

$$
\left\|\widehat{u}(x, \cdot)-\widehat{u}_{c}(x, \cdot)\right\| \leq C\left(e^{-x \sqrt{\xi_{c}^{2}+k^{2}}}\|\widehat{u}(0, \cdot)\|+e^{-(L-x) \sqrt{\xi_{c}^{2}+k^{2}}}\|\widehat{u}(L, \cdot)\|\right)
$$

Thus the proof is complete.

Remark 2.5. The constant $C$ is well defined, but its value has to be estimated. From a numerical computation we conclude that $C<2$.

Remark 2.6. When solving (2.2) numerically we need Cauchy data $\left[u, u_{x}\right]=\left[g_{\delta}, h_{\delta}\right]$, along the line $x=1$. The most natural way to obtain this is to use two thermocouples, located at $x=1$, and $x=L$, and compute $h_{\delta}$ by solving a well-posed problem for the Helmholtz equation in the interval $[1, L]$. Hence it is natural to assume knowledge about $u$ at a second point.

Let us summarize what we have so far. The constant $C$ in Lemma 2.4 is unchanged. The propagated data error is estimated using Lemma 2.3:

$$
R_{b}=\left\|u_{c}-u_{c}^{\delta}\right\| \leq e^{\sqrt{\xi_{c}^{2}+k^{2}}(1-x)}\left(\left\|g-g_{\delta}\right\|+\frac{\left\|h-h_{\delta}\right\|}{\sqrt{\xi_{c}^{2}+k^{2}}}\right)
$$

and the truncation error is estimated using Lemma 2.4:

$$
R_{a}=\left\|u-u_{c}\right\| \leq C\left(e^{-x \sqrt{\xi_{c}^{2}+k^{2}}}\|u(0, \cdot)\|+e^{(x-L) \sqrt{\xi_{c}^{2}+k^{2}}}\|u(L, \cdot)\|\right)
$$

These two results can be combined into an error estimate for the spectral method. This is demonstrated in two examples.

Example 2.7. Suppose that $\|u(x, \cdot)\| \leq E$, and that we have an estimate of the noise level, $\left\|g-g_{\delta}\right\|+\left\|h-h_{\delta}\right\| \leq \delta$. If $L \geq 3$ and if we choose $\xi_{c}=\sqrt{(\log (E / \delta))^{2}-k^{2}}$, with this choice, then by expression (2.42),

$$
R_{a} \leq E e^{-x \sqrt{\xi_{c}^{2}+k^{2}}} C\left(1+e^{(2 x-L) \sqrt{\xi_{c}^{2}+k^{2}}}\right) \leq C E^{1-x} \delta^{x}\left(1+e^{-1}\right) \leq 2 E^{1-x} \delta^{x}
$$


where we have assume that $0<x<1$ and used the bound $C<2$. By expression (2.41),

$$
R_{b} \leq e^{\sqrt{\xi_{c}^{2}+k^{2}}(1-x)} \delta \leq E^{(1-x)} \delta^{x}
$$

Thus we obtain an error estimate:

$$
\left\|\widehat{u}(x, \cdot)-\widehat{u}_{c}^{\delta}(x, \cdot)\right\| \leq R_{a}+R_{b} \leq 3 E^{(1-x)} \delta^{x} .
$$

Note that, under these assumptions, $\xi_{c}=\sqrt{(\log (E / \delta))^{2}-k^{2}}$ can be used as a rule for selecting the regularization parameter.

Example 2.8. Suppose that the Helmholtz equation is valid in the interval $0 \leq x \leq 5$ and that we have a priori bounds $\|u(0, \cdot)\| \leq 1$ and $\|u(5, \cdot)\| \leq 0.5$. Furthermore, we assume that the measured data satisfies $\left\|g-g_{\delta}\right\| \leq 10^{-3}$ and $\left\|h-h_{\delta}\right\| \leq 0.5 \cdot 10^{-2}$. Then we have the estimates:

$$
\begin{gathered}
R_{b}=\left\|u_{c}-u_{c}^{\delta}\right\| \leq e^{(1-x) \sqrt{\xi_{c}^{2}+k^{2}}}\left(10^{-3}+0.5 \cdot \frac{10^{-2}}{\sqrt{\xi_{c}^{2}+k^{2}}}\right), \\
R_{a}=\left\|u-u_{c}\right\| \leq 2\left(e^{-x \sqrt{\xi_{c}^{2}+k^{2}}} \cdot 1+e^{(x-5) \sqrt{\xi_{c}^{2}+k^{2}}} \cdot 0.5\right) .
\end{gathered}
$$

By balancing these two components $R_{a}$ and $R_{b}$, we can find a suitable value for the regularization parameter.

\section{Numerical Implementation}

In this section, we use the method of Lines. Let

$$
\begin{gathered}
\mathbf{U}=U(x)=\left(\begin{array}{c}
U_{0}(x) \\
U_{1}(x) \\
U_{2}(x) \\
\vdots \\
U_{n}(x)
\end{array}\right) \approx\left(\begin{array}{c}
U\left(x, y_{0}\right) \\
U\left(x, y_{1}\right) \\
U\left(x, y_{2}\right) \\
\vdots \\
U\left(x, y_{n}\right)
\end{array}\right), \\
y_{i}=\frac{i}{n}, \quad i=0,1,2, \ldots, n,
\end{gathered}
$$




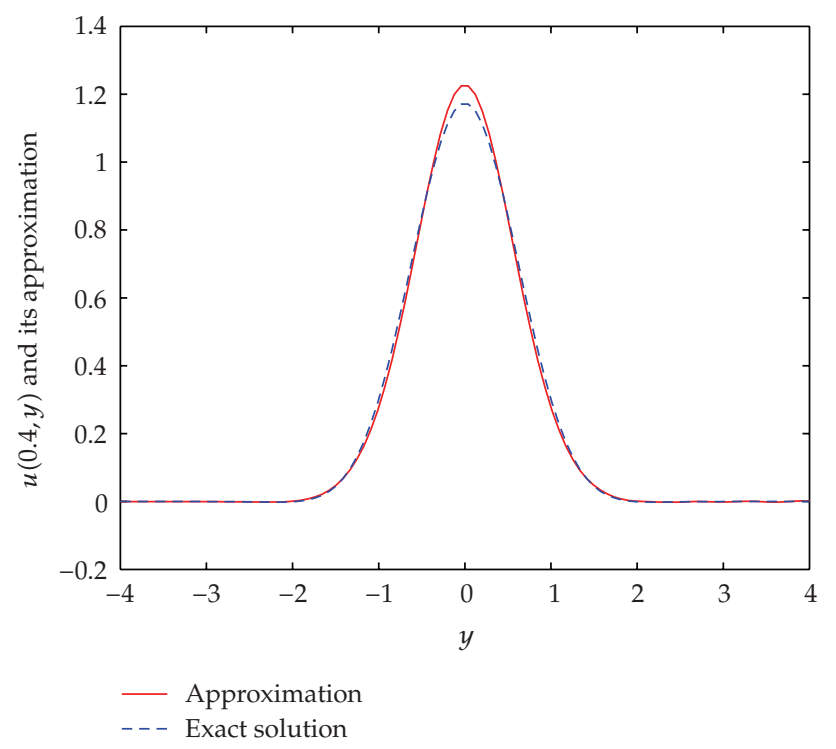

Figure 1: $x=0.4, \delta=1 * 10^{-5}, \xi_{c}=11.35$.

and then we can obtain

$$
\begin{gathered}
\left(\begin{array}{c}
\mathbf{U}_{x} \\
\mathbf{U}
\end{array}\right)_{x}=\left(\begin{array}{ll}
\mathbf{0} & \mathbf{D} \\
\mathbf{I} & \mathbf{0}
\end{array}\right)\left(\begin{array}{l}
\mathbf{U}_{x} \\
\mathbf{U}
\end{array}\right), \\
\left(\begin{array}{c}
\mathbf{U}_{x}(\mathbf{1}) \\
\mathbf{U}(\mathbf{1})
\end{array}\right)=\left(\begin{array}{l}
\mathbf{H} \\
\mathbf{G}
\end{array}\right),
\end{gathered}
$$

where

$$
\begin{array}{r}
\mathbf{H}=\left(\begin{array}{c}
u_{x}\left(1, y_{0}\right) \\
u_{x}\left(1, y_{1}\right) \\
u_{x}\left(1, y_{2}\right) \\
\vdots \\
U_{x}\left(1, y_{n}\right)
\end{array}\right), \\
\mathbf{G}=\left(\begin{array}{c}
U\left(1, y_{0}\right) \\
U\left(1, y_{1}\right) \\
U\left(1, y_{2}\right) \\
\vdots \\
U\left(1, y_{n}\right)
\end{array}\right) .
\end{array}
$$




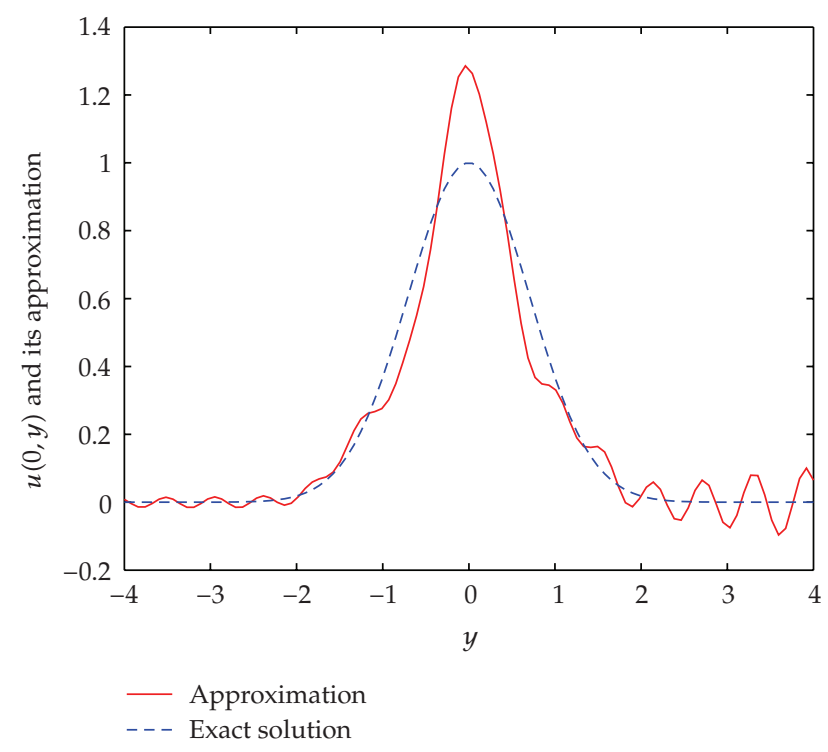

Figure 2: $x=0, \delta=1 * 10^{-5}, \xi_{c}=11.35$.

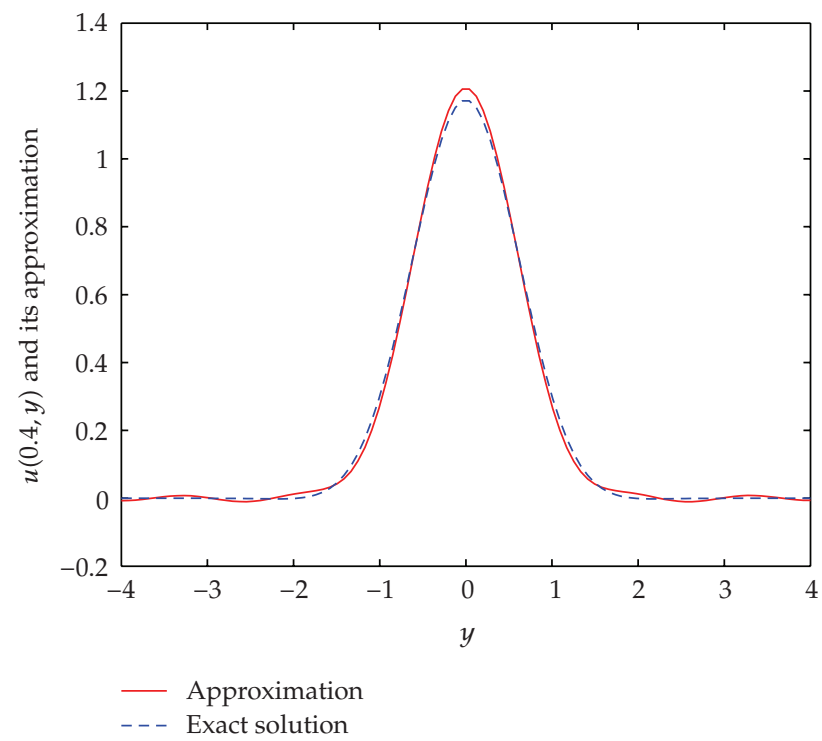

Figure 3: $x=0.4, \delta=1 * 10^{-2}, \xi_{c}=4.45$.

The matrix $D$ approximates the second-order derivative $u_{y y}$ by spectral cutoff method (see [2]). This ordinary differential equation system can easily be solved by various numerical methods.

Example 3.1. It is easy to verify that the function $u(x, y)=\sin x \cosh \left(\sqrt{1+k^{2}} y\right) /\left(5+k^{2}\right)$ is the exact solution of (1.2). Now we need to seek the solution $u(x, y)=\sin x \cosh \left(\sqrt{1+k^{2}} y\right) /(5+$ $\left.k^{2}\right)$, where $0 \leq x<1$ from the Cauchy data pairs $u(1, y)=\sin 1 \cosh \left(\sqrt{1+k^{2}} y\right) /\left(5+k^{2}\right)$ and $u_{x}(1, y)=\cos 1 \cosh \left(\sqrt{1+k^{2}} y\right) /\left(5+k^{2}\right)$. 


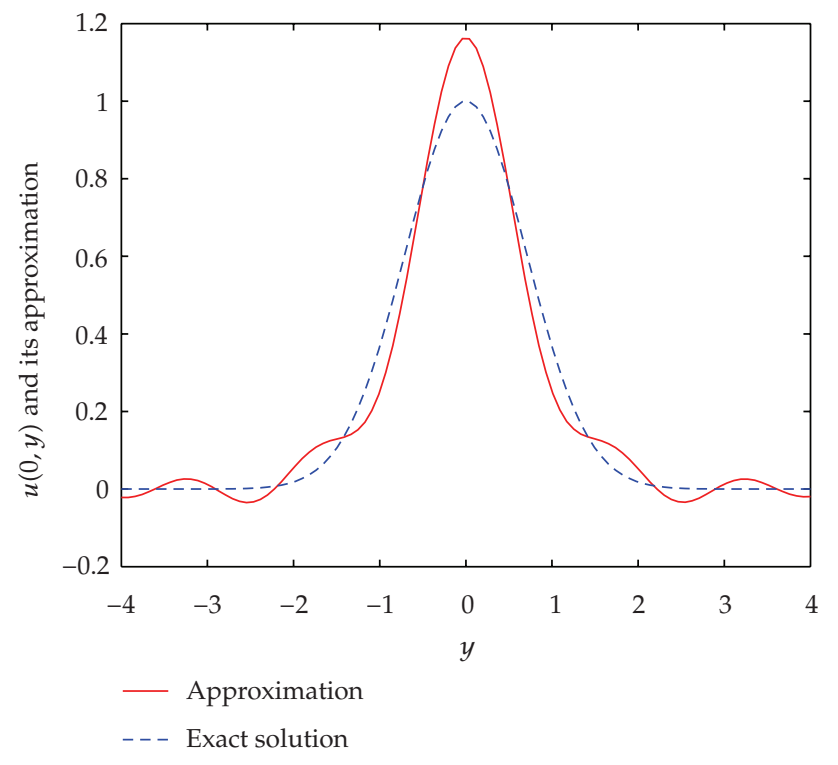

Figure 4: $x=0, \delta=1 * 10^{-2}, \xi_{c}=4.45$.

In our numerical experiment, we give the comparison between the exact solution of problem (2.2) and its approximation by spectral cutoff method for different noise levels $\delta$ and different locations $x$. Please see Figures 1, 2, 3, and 4 . In the experiment, the regularization parameter is chosen according to the Remark of Remark 2.5, where we take $E=1$. From the above results, it is easy to see that the numerical effect of the spectral method works well. Moreover, we can also see that the lower the noise level $\delta$ is, the better the approximate effect is; the closer to the boundary $x=1$ the location is, the better the approximate effect is. These accord with the theory in Section 2.

\section{Acknowledgments}

The author wants to express his thanks to the referee for many valuable comments. This work is supported by the Educational Commission of Hubei Province of China (Q20102804, T201009).

\section{References}

[1] A. Kirsch, An Introduction to the Mathematical Theory of Inverse Problems, vol. 120 of Applied Mathematical Sciences, Springer, New York, NY, USA, 1996.

[2] I. Harari, P. E. Barbone, M. Slavutin, and R. Shalom, "Boundary infinite elements for the Helmholtz equation in exterior domains," International Journal for Numerical Methods in Engineering, vol. 41, pp. 1105-1131, 1998.

[3] L. Marin, L. Elliott, P. J. Heggs, D. B. Ingham, D. Lesnic, and X. Wen, "An alternating iterative algorithm for the Cauchy problem associated to the Helmholtz equation," Computer Methods in Applied Mechanics and Engineering, vol. 192, no. 5-6, pp. 709-722, 2003.

[4] L. Marin, L. Elliott, P. J. Heggs, D. B. Ingham, D. Lesnic, and X. Wen, “Conjugate gradient-boundary element solution to the Cauchy problem for Helmholtz-type equations," Computational Mechanics, vol. 31, no. 3-4, pp. 367-377, 2003. 
[5] T. Regińska and K. Regiński, "Approximate solution of a Cauchy problem for the Helmholtz equation," Inverse Problems, vol. 22, no. 3, pp. 975-989, 2006.

[6] L. Marin, L. Elliott, P. J. Heggs, D. B. Ingham, D. Lesnic, and X. Wen, "Comparison of regularization methods for solving the Cauchy problem associated with the Helmholtz equation," International Journal for Numerical Methods in Engineering, vol. 60, no. 11, pp. 1933-1947, 2004.

[7] J. Hadamard, Lectures on the Cauchy Problem in Linear Partial Differential Equations, Yale University Press, New Haven, Conn, USA, 1923.

[8] X. T. Xiong and C. L. Fu, "Two approximate methods of a Cauchy problem for the Helmholtz equation," Computational \& Applied Mathematics, vol. 26, no. 2, pp. 285-307, 2007.

[9] C. L. Fu, X. L. Feng, and Z. Qian, "The Fourier regularization for solving the Cauchy problem for the Helmholtz equation," Applied Numerical Mathematics, vol. 59, no. 10, pp. 2625-2640, 2009.

[10] H. H. Qin and T. Wei, "Modified regularization method for the Cauchy problem of the Helmholtz equation," Applied Mathematical Modelling, vol. 33, no. 5, pp. 2334-2348, 2009

[11] A. L. Qian, X. T. Xiong, and Y. J. Wu, "On a quasi-reversibility regularization method for a Cauchy problem of the Helmholtz equation," Journal of Computational and Applied Mathematics, vol. 233, no. 8, pp. 1969-1979, 2010

[12] L. Eldén and F. Berntsson, "Spectral and wavelet methods for solving an inverse heat conduction problem," in Inverse Problems in Engineering Mechanics, pp. 3-10, Elsevier, Oxford, UK, 1998.

[13] F. Berntsson, "A spectral method for solving the sideways heat equation," Inverse Problems, vol. 15, no. 4, pp. 891-906, 1999.

[14] X. T. Xiong, C. L. Fu, and J. Cheng, "Spectral regularization methods for solving a sideways parabolic equation within the framework of regularization theory," Mathematics and Computers in Simulation, vol. 79 , no. 5, pp. 1668-1678, 2009. 